\title{
Simulación del comportamiento térmico en exteriores urbanos correlacionando las variables de calor antropogénico vehicular y orientación
}

\section{Simulation of thermal behavior in urban exteriors correlating the variables of vehicular anthropogenic heat and orientation}

GRAJEDA-ROSADO, Ruth María†*, ALONSO-GUZMAN, Elia Mercedes, ESPARZA-LOPEZ, Carlos Javier y ESCOBAR-DEL POZO, Carlos

Universidad Michoacana de San Nicolas de Hidalgo-Programa Interinstitucional de Doctorado, Morelia, México Universidad Veracruz, Veracruz, México

ID $1^{\text {er }}$ Autor: Grajeda-Rosado, Ruth María / ORC ID: 0000-0001-9961-3541 CVU CONACYT ID: 802541

ID $1^{\text {er }}$ Coautor: Alonso-Guzman, Elia Mercedes / ORC ID: 0000-0002-8502-4313, Researcher ID Thomson: B-8744-2018, CVU CONACYT ID: 26331

ID $2^{\text {do }}$ Coautor: Esparza-Lopez, Carlos Javier / ORC ID: 0000-0003-0058-5072, Researcher ID Thomson: G-8108-2018, CVU CONACYT ID: 335287

ID $3^{\text {er }}$ Coautor: Escobar-Del Pozo, Carlos / ORC ID: 0000-0002-8732-8791, Researcher ID Thomson: G-8111-2018, CVU CONACYT ID: 43347

\section{Resumen}

La complejidad del entorno urbano y su innegable nexo con la ecuación del balance energía, abre las puertas a los investigadores para comprender fenómenos como la Isla de Calor Urbano (ICU). Un factor poco estudiado de la ICU es el calor antropogénico vehicular. Mediante el software Computacional Fluid Dynamics (CFD), se busca comprender el entorno térmico dentro de un cañón urbano, en función de dos variables, el calor producido por la máquina de combustión de los vehículos y la orientación de la calle (Norte - Sur y Este - Oeste). El análisis de la correlación de estas variables se basa en la información obtenida de la simulación en diferentes estratos de altura, considerando valores constantes como la radiación directa, radiación difusa, emisividad y absortividad de los materiales y una temperatura fija para el capó o cofre del auto, alterando la cantidad de vehículos automotores y la orientación para examinar los diferentes patrones de los perfiles térmicos. La investigación abre la pauta para comprender dicho fenómeno y ser considerado en las simulaciones para la eficiencia energética de los edificios, puesto que impacta directamente en las fachadas de las construcciones y en la determinación de las técnicas pasivas y activas de enfriamiento.

Simulación, Calor antropogénico vehicular, Cañón urbano

\begin{abstract}
The complexity of the urban environment and its undeniable connection with the energy balance equation opens the doors for researchers to understand phenomena such as the Urban Heat Island (UHI). The least studied factor of the UHI is vehicular anthropogenic heat. Using Computational Fluid Dynamics (CFD) software, the aim is to understand the thermal environment within an urban canyon, based on two variables, the heat produced by the vehicle combustion engine and the orientation of the street (North - South and East -West). The analysis of the correlation of these variables is based on the information obtained from the simulation in different height strata, considering constant values such as direct radiation, diffuse radiation, emissivity and absorptivity of materials and a fixed temperature for the bonnet or hood of the car, altering the number of automotive vehicles and the orientation to examine the different patterns of the thermal profiles. The research opens the way to understand this phenomenon and be considered in simulations for the energy efficiency of buildings, since it directly impacts the facades of buildings and the determination of passive and active cooling techniques.
\end{abstract}

Simulation, Vehicular anthropogenic heat, Urban canyon

Citación: GRAJEDA-ROSADO, Ruth María, ALONSO-GUZMAN, Elia Mercedes, ESPARZA-LOPEZ, Carlos Javier y ESCOBAR-DEL POZO, Carlos. Simulación del comportamiento térmico en exteriores urbanos correlacionando las variables de calor antropogénico vehicular y orientación. Revista de Simulación y Laboratorio. 2019, 6-21: 19-33

*Correspondencia al Autor (Correo electrónico: lgrajeda@uv.mx - ruthgrajeda10@yahoo.com.mx)

$\dagger$ Investigador contribuyendo como primer Autor 


\section{Introducción}

Desde casi un siglo, se sabe que los asentamientos urbanos muestran un incremento de temperatura en relación con sus alrededores rurales, fenómeno ampliamente reportados en diferentes ciudades del mundo y conocido como Isla de Calor Urbano.

Además, los investigadores reportan problemáticas asociadas con la Isla de Calor Urbano (ICU) como, el aumento de uso de energía ligado con la generación de gases de invernadero y potencialización en la mortalidad por estrés a causa del aumento de temperatura que resienten los habitantes de manera más prolongada y que en algunos casos, se convierte en ola de calor (Li et al, 2018).

Varios son los factores que alteran el clima en la ciudad y causan la formación de las isotermas de la ICU: a) las propiedades físicotérmicas de las superficies urbanas (reflectancia solar, albedo, emisividad, emitancia), b) la alteración del porcentaje de evapotranspiración asociada con los metros cuadrados de áreas verdes, c) variables de diseño urbano (densidad de construcción, uso de suelo, geometría urbana o relaciones de aspecto, orientación y factor de vista del cielo y por último, pero no menor d) el calor antropogénico $\left(\mathrm{Q}_{\mathrm{F}}\right)$, de los seres humanos, industria, edificios y vehículos (Santamouris et al, 2011).

Este último, Calor Antropogénico Vehicular $\left(\mathrm{Q}_{\mathrm{FV}}\right)$ es el tema asociado con la presente investigación; factor estudiado de manera global con los otros flujos de calor $\mathrm{Q}_{\mathrm{F}}$, debido a su complejidad en la toma de mediciones en campo y la diversidad de variables que intervienen en él.

La unidad de análisis tomada en este caso fue el cañón, estructura urbana que comparte similitudes aun en diferentes ciudades, como es su ubicación horizontal (microescala) y vertical (dosel). Dentro de la contextualización de la ICU, el cañón urbano se pude puntualizar como un microclima generado por las diversas configuraciones urbanas dentro de la capa de dosel y afectado por su entorno inmediato, su geometría y propiedades físicas-térmicas de los materiales (Santamouris et al, 2011) (Strommann-Andersen et al, 2011).
El primer acercamiento del flujo de calor, QF; fue la consideración de sinónimo de consumo de energía (Oke, 1988) donde, su incorporación en los modelos de simulación de clima urbano es relativamente sencilla, incluido generalmente como constantes, en las ecuaciones presupuestarias de energía de volumen de superficie y de control.

$\mathrm{QF}=\mathrm{Q}_{\mathrm{FV}}+\mathrm{Q}_{\mathrm{FH}}+\mathrm{Q}_{\mathrm{FM}}$

Donde:

$\mathrm{QFv}_{\mathrm{F}}$ Calor liberado por vehículos.

$\mathrm{Q}_{\mathrm{FH}}=$ Calor liberado por edificaciones $\mathrm{e}$ industria.

$\mathrm{QFM}_{\mathrm{FM}}$ Calor liberado por el metabolismo humano.

Una manera de determinar la magnitud de $\mathrm{Q}_{\mathrm{F}}$ es correlacionarla con la densidad de población. A escala continental, este valor es bajo en magnitud, $0.4 \mathrm{Wm}^{-2}$ en EEUU, $0.7 \mathrm{Wm}^{-}$ 2 en Europa Occidental y $0.2 \mathrm{Wm}^{-2}$ (China) (Flanner, 2009). Pero dentro de las escalas menores como una urbe, los investigadores han estimado emisiones de $\mathrm{Q}_{\mathrm{F}}$ del orden de 10-100 $\mathrm{Wm}^{-2}$ para ciudades tan diversas como Lodz, Polonia y Philadelphia, EE.UU. (Sailor et al, 2015). En una escala menor como una manzana, Ichionse (1999), lo asocia proporcionalmente con la altura de los edificios (número de pisos) dando valores de $400 \mathrm{Wm}^{-2}$ como promedio durante el día y ascendiendo a $1590 \mathrm{Wm}^{-2}$ durante el invierno en el centro de Tokio. Por lo tanto, se dice que su magnitud es inversamente proporcional a su escala de estudio, es decir, en microescala es más alta, mientras que disminuye cuando se promedia con los valores de una ciudad (Stewart et al, 2012) (Figura 1).

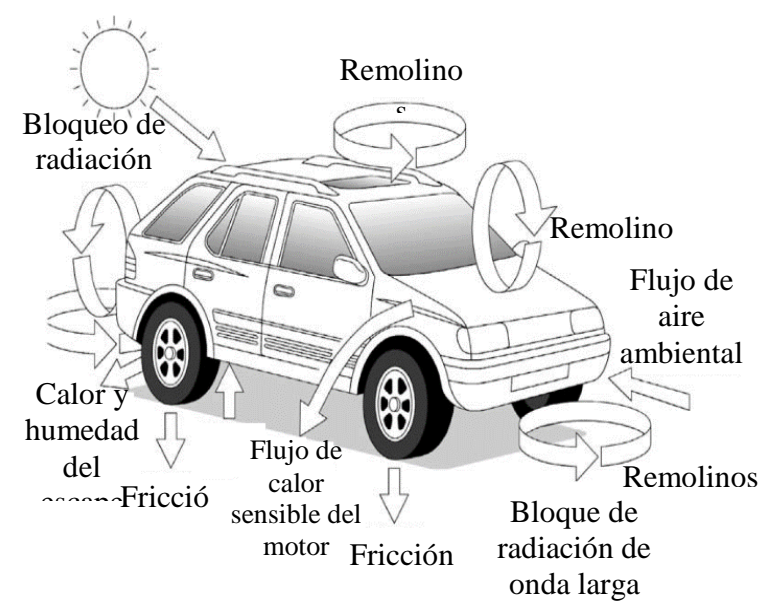

Figura 1 Impacto de los vehículos sobre la temperatura de las superficies de rodamiento

Fuente:(Chapman et al, 2005) 
La importancia de considerar $\mathrm{QF}_{\mathrm{F}}$, es mostrada en algunos estudios que confirman un aumento en la temperatura del aire, dentro de la urbe de 1 a $2^{\circ} \mathrm{C}$ en verano y 2 a $3^{\circ} \mathrm{C}$ en verano (Fan et al, 2005), gracias a este factor.

Koralegedara, et al, (2016), reportan que hay cuatro métodos para su cuantificación los cuales son a) enfoque basado en inventarios ascendentes, b) enfoque basado en inventarios descendente, c) modelado del balance de energía y d) regresión estadística. Las presentes metodologías presenten sus resultados en $\mathrm{Wm}^{-2}$.

Las dos primeras metodologías usan datos de consumo de energía con resolución espacial y temporal, $\mathrm{Q}_{\mathrm{FH}}$ usa valores oficiales de instituciones o determina una cantidad de acuerdo con el tipo de edificación de la zona de estudio, QFv monitorea cantidades como el tráfico por día u hora, los tipos de vehículos y las propiedades del consumo de energía del combustible, ambos métodos requieren una tediosa adquisición de datos y tienen complicaciones prácticas en las mediciones en campo (Sailor D. , 2011).

Una ecuación propuesta por expertos como Sailor y Lu (2004), para el cálculo de QFV por el método de inventarios ascendentes es:

$\mathrm{QFV}_{\mathrm{FV}}=\operatorname{pcDVDFt}(\mathrm{h}) \rho_{\mathrm{pop}}(\mathrm{h}) \mathrm{EV}$

Donde:

$\mathrm{Ft}=$ Perfiles de tráfico por hora.

$\mathrm{pcDVD}=$ Distancia diaria per cápita por vehículo.

$\rho_{\text {pop }}(\mathrm{h})=$ Densidad de población por hora.

$\mathrm{EV}=$ Liberación de energía por vehículo por metro de recorrido

La presente investigación explora y analiza a través de la simulación con el software Computational Fluid Dynamics (CFD) relacionar el perfil térmico del cañón urbano y el calor expulsado por los vehículos, en función de los parámetros geométricos o relaciones de aspecto de la calle $(\mathrm{H}=$ alto de los edificios entre $\mathrm{W}=$ ancho de la superficie de rodamiento) como propuesta urbana para modificar el fenómeno de la ICU. Se agrega la variable de orientación de la vialidad, como punto de análisis, porque los resultados finales demuestran que es un factor determinante para el comportamiento de la temperatura interna de la calle.
Con los datos obtenidos, se comprueba que la temperatura del aire dentro del cañón, se incrementa $2^{\circ} \mathrm{C}$ por cada 10 autos más que transitan en él, pero esto puede aumentar si la orientación es Norte-Sur (fachadas orientadas al Este-Oeste).

El artículo, primeramente, introduce al lector en la problemática del calor antropogénico $\left(Q_{F}\right)$ y su importancia en su consideración, a la hora de diseñar espacios urbanos y determinar su aportación de carga térmica al establecer el consumo de energía de los edificios, posteriormente, el apartado dos, describe la geometría del modelo de simulación y sus condiciones de frontera, la sección tres explica las mediciones de campo que permiten la validación de los resultados. Los datos y resultados obtenidos son analizados en graficas para finalizar con las conclusiones.

\section{Descripción del modelo}

Los métodos para la predicción de las temperaturas del aire urbano en sus diferentes escalas horizontales y verticales, según Kolokotroni et al. (2010), se clasifican en cuatro categorías: a) modelos de climatología; b) modelos empíricos; c) modelos de dinámica de fluidos computacional (CFD); y d) métodos de regresión estadística. En general, según Mirzaei (2015), el impacto de diferentes parámetros tales como: a) la orientación del edificio, b) relación de aspecto, c) materiales de superficie y d) la vegetación en el cálculo de la convección superficial, valoraciones térmicas para la comodidad de los peatones, y la ventilación urbana puede ser investigado usando CFD.

En este caso, los cálculos de transferencia de calor se resolvieron con el ANSyS FLUENT, debido a su versatilidad como una herramienta para predecir, con cierta certeza, el comportamiento de los fluidos sobre diversas superficies, figuras y ambientes en condiciones estables, inestables, isotérmicos, reactivos, compresibles e incompresibles. Para generar los datos, se requiero idealizar el cañón urbano, mediante un prisma rectangular, del cual se substraen volúmenes que simulan las cuadras y los coches, el volumen final de aire quedo de 190 metros de largo por 180 metros de ancho, con una altura de 60 metros, que es tres veces más la altura del edificio mayor, permitiendo que el viento se comporte de manera estable en la parte superior del volumen (Figura 2). 


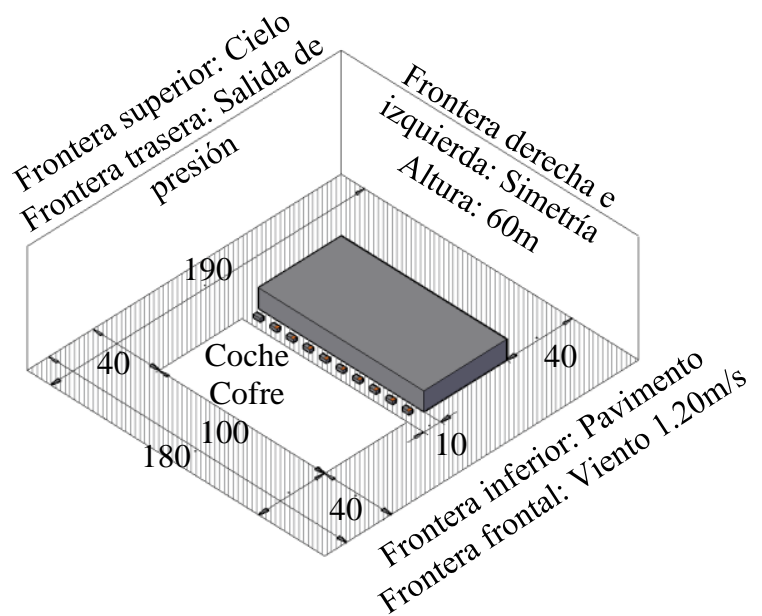

Figura 2 Isométrico del dominio computacional, con dimensiones, radio de aspecto $=1$ y colocación de 10 autos Fuente: Elaboración Propia

Los materiales usados en las simulaciones fueron: lámina de acero en la carrocería del auto, concreto en pavimento y el aplanado de cemento:arena en las paredes de los edificios; estos dos últimos debido a la similitud de materiales usados en el caso de estudio (Calle de Independencia) que permitan su comparación con la simulación. La definición de sus espesores, como características físico-térmicas se observan en la Tabla 1.

\begin{tabular}{|l|r|r|r|}
\cline { 2 - 4 } \multicolumn{1}{c|}{} & \multicolumn{1}{c|}{$\begin{array}{c}\text { Pavi- } \\
\text { mento }\end{array}$} & \multicolumn{1}{c|}{ Pared } & Carrocería \\
\hline Material & Concreto & Aplanado & Acero \\
\hline Espesor mm & 200 & 150 & 2 \\
\hline $\begin{array}{l}\text { Densidad } \\
\mathrm{kg} / \mathrm{m}^{3}\end{array}$ & 2,300 & 1,570 & 7,850 \\
\hline $\begin{array}{l}\text { Calor especifico } \\
\text { (j/kg-k) }\end{array}$ & 840 & 1,000 & 456 \\
\hline $\begin{array}{l}\text { Conductividad } \\
\text { térmica } \\
(\mathrm{W} / \mathrm{m}-\mathrm{k})\end{array}$ & 1.00 & 0.53 & \\
\hline Emisividad & 0.90 & 0.90 & 50 \\
\hline
\end{tabular}

Tabla 1 Propiedades de los materiales usados en la simulación

Fuente: (Cengel et. al, 2011)

La cuadricula hexagonal se aplica para la simulación en Fluent, con el principio: todo lo ingresado en cada celda de la malla, es transferido en la celda adyacente, conservando la masa y el momento lineal (ANSYS, Inc., 2013). El mallado final quedo establecido con un 720 mil elementos $o$ células tetraédricas (aproximadamente), los volúmenes mínimos y máximos de las células en el dominio o volúmenes de control son de aproximadamente $0.15 \mathrm{~m}$ y $1.0 \mathrm{~m}$.
La configuración seleccionada se basa en su perfecta adaptación a la geometría del dominio de la simulación, un ejemplo lo podemos encontrar con (Nazarian et al, 2015) y (2016). La resolución de la cuadricula paso por un análisis de sensibilidad, considerando una calidad gruesa a fina $(120,000$ hasta $1,680,000$ celdas), donde los resultados tienen una diferencia menor al $1 \%$. Las iteraciones usadas fueron 500 para converger en la solución o tener residuos menores a la $10^{-3}$ en los valores según el criterio dado en la teoría del ANSyS (2013).

La ecuación base usada es el Reynolds Averaged Navier-Stokes (RANS), que obtiene el balance de energía (ec. 3) en un ambiente tridimensional, incluyendo esfuerzos viscosos y acoplados entre sí, que a través de parámetros y modelos de transferencia no afecten su continuidad, sin embargo, se busca su simplificación, imponiendo condiciones de contorno que no varíen con el tiempo (estacionarias).

$(1-\alpha) S \downarrow-L=Q_{c^{-}} Q_{h}$

$L_{g r}=\epsilon\left[\sigma T^{4}{ }_{g r}-L_{\text {inc.gr }}\right]$

$L_{\text {inc.gr }}=\left(F_{\text {sky-gr }}\right) \sigma T^{4}{ }_{\text {sky }}-L_{w-g r}$

Donde:

$L=$ Flujo neto de radiación de onda larga.

$S=$ Flujo neto de radiación de onda corta

$Q_{C}=$ Conducción.

$Q_{h}=$ Convección

$\alpha=$ Albedo superficial

$\epsilon=$ Emisividad superficial

$\sigma=$ Constante de Stephan-Boltzmann

$g r=$ Superficie del suelo

${ }_{w-g r}=$ De las paredes al suelo

$L_{i n c . g r}=$ Total de radiación larga incidente en una superficie

$T=$ Temperatura

Para el tratamiento de paredes cercanas, se utiliza el comando de Standard Wall Function, basado en el modelo de $k$-epsilon Realizable. La radiación solar fue calculada por el modelo de ANSyS Solar Load Model, el día 20 de mayo a las 13 horas. Los resultados calculados de los valores de radiación en el modelo fueron: directa $884.78 \mathrm{Wm}^{-2}$, difusa en superficies verticales de $72.57 \mathrm{Wm}^{-2}$, difusa en superficies horizontales $118.56 \mathrm{Wm}^{-2}$ y reflejada por el suelo de 99.58 $\mathrm{Wm}^{-2}$, equiparables a los valores con la localización de las mediciones en campo (Veracruz, Veracruz, México). 
Las condiciones de frontera en el modelo se muestran en la Tabla 2:

\begin{tabular}{|c|c|c|}
\hline Frontera & Condición & Comentario \\
\hline Superior & $\begin{array}{l}\text { Condición de } \\
\text { simetría } \\
\text { (Cielo) }\end{array}$ & $\begin{array}{l}\text { Para tener un perfil de } \\
\text { viento estable en la } \\
\text { parte superior del } \\
\text { modelo }\end{array}$ \\
\hline Laterales & $\begin{array}{l}\text { Condición de } \\
\text { simetría }\end{array}$ & $\begin{array}{l}\text { Se busca la estabilidad } \\
\text { del modelo }\end{array}$ \\
\hline $\begin{array}{l}\text { Frontal o } \\
\text { barlovento }\end{array}$ & $\begin{array}{l}\text { Temperatura a } \\
30^{\circ} \mathrm{C} \text { con un } \\
\text { viento } \\
1.2 \mathrm{~m} / \mathrm{s}\end{array}$ & $\begin{array}{l}\text { Consideraciones del } \\
\text { ambiente urbano, con } \\
\text { temperatura y viento } \\
\text { promedio en la ciudad } \\
\text { de estudio. }\end{array}$ \\
\hline $\begin{array}{l}\text { Posterior o } \\
\text { sotavento }\end{array}$ & $\begin{array}{l}\text { Temperatura } \\
\text { de salida a } \\
30^{\circ} \mathrm{C}\end{array}$ & $\begin{array}{l}\text { Para inducir una salida } \\
\text { de viento }\end{array}$ \\
\hline $\begin{array}{l}\text { Inferior } \mathrm{o} \\
\text { pavimento }\end{array}$ & Pared & $\begin{array}{l}\text { Información de las } \\
\text { propiedades }\end{array}$ \\
\hline Edificios & Pared & 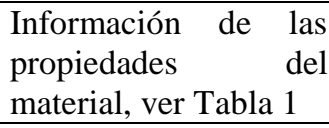 \\
\hline Coches & Pared & $\begin{array}{ll}\text { Información de las } \\
\text { propiedades }\end{array}$ \\
\hline Cofre & $\begin{array}{l}\text { Pared a una } \\
\text { temperatura } \\
\text { constante de } \\
60^{\circ} \mathrm{C}\end{array}$ & $\begin{array}{l}\text { Información de las } \\
\text { propiedades }\end{array}$ \\
\hline
\end{tabular}

Tabla 2 Condiciones de frontea Fuente: Elaboración Propia

Los 900 datos de cada simulación fueron obtenidos de nueve líneas a lo largo del cañón, ubicadas a 1.5 metros de las fachadas y en medio del cañón, con varias alturas, 1.50, 3.0 y $4.5 \mathrm{~m}$ en perpendicular al suelo (Figura 3 ).

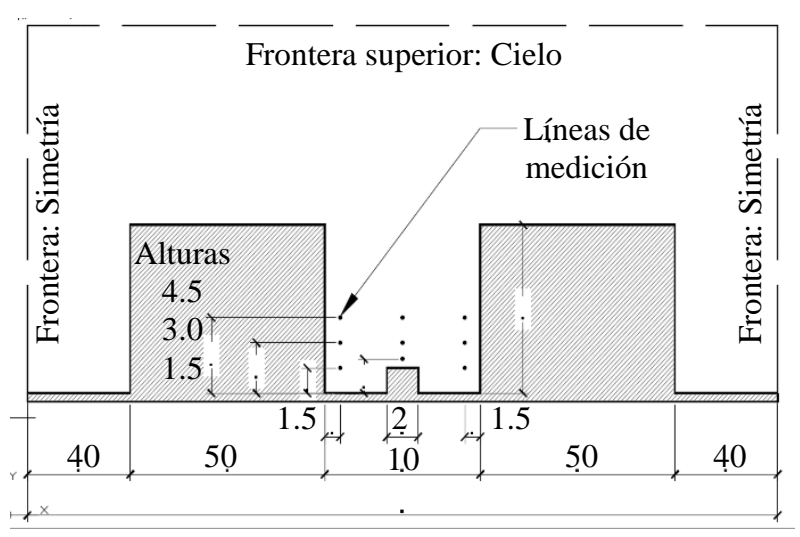

Figura 3 Sección con ubicación de las nueve líneas de medición para la toma de valores en la simulación Fuente: Elaboración Propia

\section{Metodología}

Basándose en Hernández Sampieri et al. (2014), el presente documento se define con dos tipos de alcances, correlacionar y explicar.

Por lo tanto, estos alcances tienen como finalidad conocer el grado de asociación que existe entre más de dos categorías, en este caso particular, las variables independientes son: el número de autos, la orientación, las condiciones climáticas y las relaciones geométricas de una calle con la variable dependiente, la temperatura interna del cañón (contexto especifico) (Figura 4). Para lograr con esto, responder y explicar cómo es afectado el perfil térmico y como modificarlo a favor de las necesidades de los habitantes.

Un objetivo fundamental de la investigación fue el diseño de una metodología que permita recolectar datos en campo, para encontrar el valor $\mathrm{Q}_{\mathrm{FV}}$ a microescala dentro de un cañón urbano en valores de ${ }^{\circ} \mathrm{C}$, el cual permitió ser comparado con los valores arrojados en las simulaciones ejecutadas por el software ANSyS y aportar valores de referencia para entender el fenómeno.

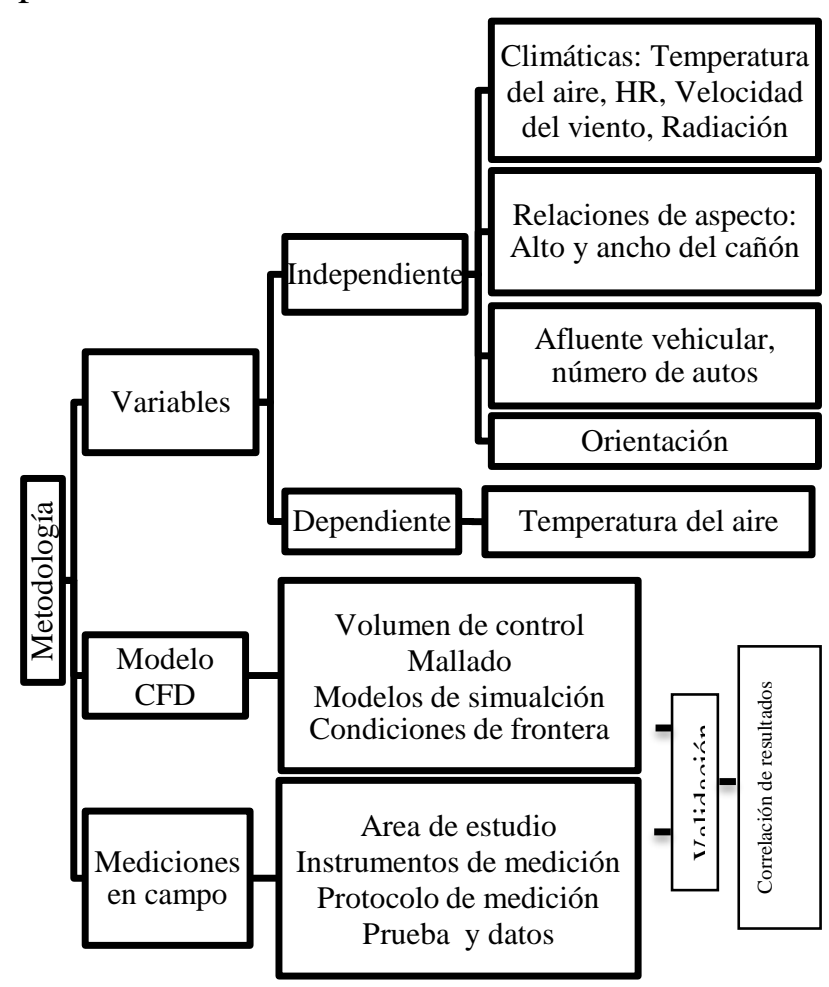

Figura 4 Metodología de la investigación Fuente: Elaboración Propia 
El método, diseñado a partir del marco teórico, es definido como no experimental con diseño transaccional. Fundamentado por la definición de Hernández et al. (2014) es clasificando por su dimension temporal o el número de momentos o puntos en el tiempo en los cuales se recolectan datos en un lapso ininterrupido, esto se logró usando un dron. Con ello, la metodología de mediciones con vuelo automático, es decir, dron; permitió:

- Analizar cuál es el nivel de modificación de la variable dependiente en un momento dado; y

- Evaluar el fenómeno en un punto del tiempo.

Analizando diferentes estudios con metodologías basadas en mediciones en campo para el análisis de otras variables, se adecua el procedimiento para su aplicación en una microescala, calle; poniendo a prueba los alcances del desplazamiento autónomo de drones para áreas abiertas, aplicándolos en la recolección de variables ambientales y lograr la validación de los resultados en las simulaciones.

\section{Validación}

Las mediciones "in situ" son una metodología para determinar la validez predictiva de un modelo y definir de manera asertiva las variables dependientes, que en esta investigación intervienen en los intercambios de flujo de calor a nivel urbano.

La comparación de valores de simulación se basa en las campañas realizadas en campo en la ciudad de Veracruz, ubicada en el golfo de México (latitud-norte $19^{\circ} 10^{\prime}$ y longitud-oeste $\left.96^{\circ} 08^{\prime}\right)$, en la calle de Independencia en el centro de la ciudad (Figura 5).

La pertinencia de realizar las mediciones en esta área urbana es porque la calle tiene 10 metros de ancho y los edificios en promedio tiene 10 metros de alto, además el tráfico solo es compuesto por autos privados, no es una calle transitada por autobuses o camiones (Figura 6).

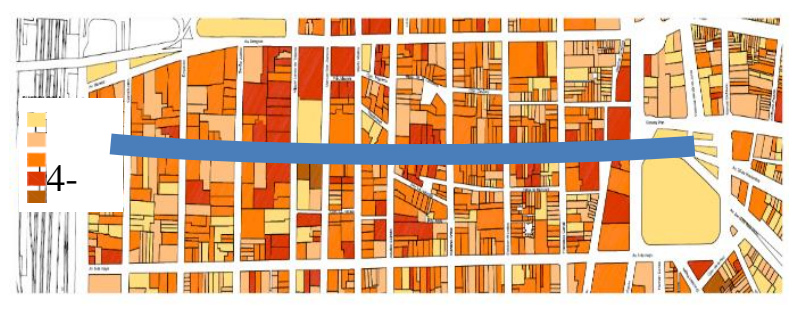

Figura 6 Vista en planta de la calle en una sección del centro de Veracruz y área de estudio de la investigación Fuente: Modificado de plano oficial de la Dirección de Desarrollo Urbano y Ordenamiento Territorial

Los días que se tomaron las medias fueron el 19 y 20 de mayo del 2018, sábado y domingo; como es zona comercial el afluente vehicular se modifica durante el fin de semana, siendo el sábado el día más concurrido.

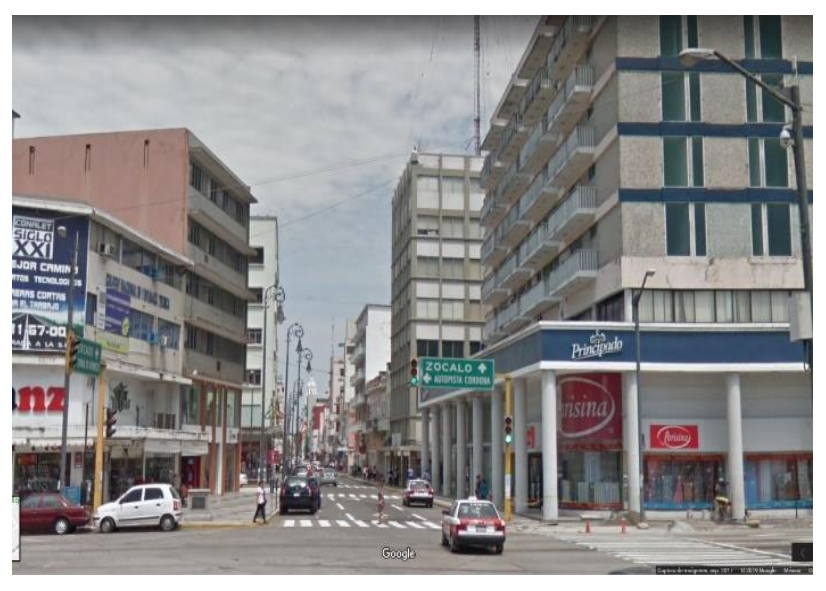

Figura 5 Fotografía de la calle de Independencia Fuente: Googlemaps

En ambas fechas se presentó una temperatura promedio de $32^{\circ} \mathrm{C}$ y una humedad relativa del $70 \%$ en la ciudad. Dentro de las condiciones presentadas en campo, la velocidad aproximada de los autos fue de $40 \mathrm{~km} / \mathrm{hr}$. La recolección inicio a las 13:30 horas, sin embargo, durante el proceso, la fachada oriente presentaba sombra y el poniente una exposición solar de 30 minutos.

Para la recolección de datos se usó el sensor de temperatura y humedad SHT7 con una precisión de $\mathrm{T}= \pm 0.3^{\circ} \mathrm{C}$ y $\mathrm{HR}= \pm 1.8 \%$, resolución de $\mathrm{T}=0.01^{\circ} \mathrm{C}$ y $\mathrm{HR}=0.05 \%$, tiempo de respuesta $\mathrm{T}=5-30 \mathrm{sec}$ y $\mathrm{HR}=8 \mathrm{sec}$, y temperatura de operación de $-40{ }^{\circ} \mathrm{C}$ a $123.8^{\circ} \mathrm{C}$.

El sensor fue ubicado en la parte superior debido a que el dron utiliza un sistema de propulsión y sustentación tipo ala rotativa, por lo que los sensores fueron colocados arriba del mismo para no afectar las mediciones. 
Cabe destacar, que las mediciones fueron tomadas en los ejes $\mathrm{X}, \mathrm{Y}$ y $\mathrm{Z}$ al igual que las líneas colocadas en el software; para conocer más sobre la metodología referirse a (Grajeda et al, 2018).

Los valores mostrados en la Gráfica 1, las líneas continuas muestran los promedios de temperatura ( $\mathrm{T}_{\mathrm{WA}}$ ) obtenidos por las mediciones y las simulaciones en general.

Las líneas punteadas son los datos obtenidos a una altura (posición Z) de 1.50 y 3.0 metros, con variables en la posición $\mathrm{X}$, es decir, a $1.50 \mathrm{~m}$ de la fachada izquierda, en el centro del cañón y a $1.50 \mathrm{~m}$ de la fachada derecha.

Es importante aclarar, se hace la comparación con un aforo de 10 autos en la simulación y 12 autos en las mediciones de campo, es decir, datos tomados el sábado 19 de mayo del 2018.

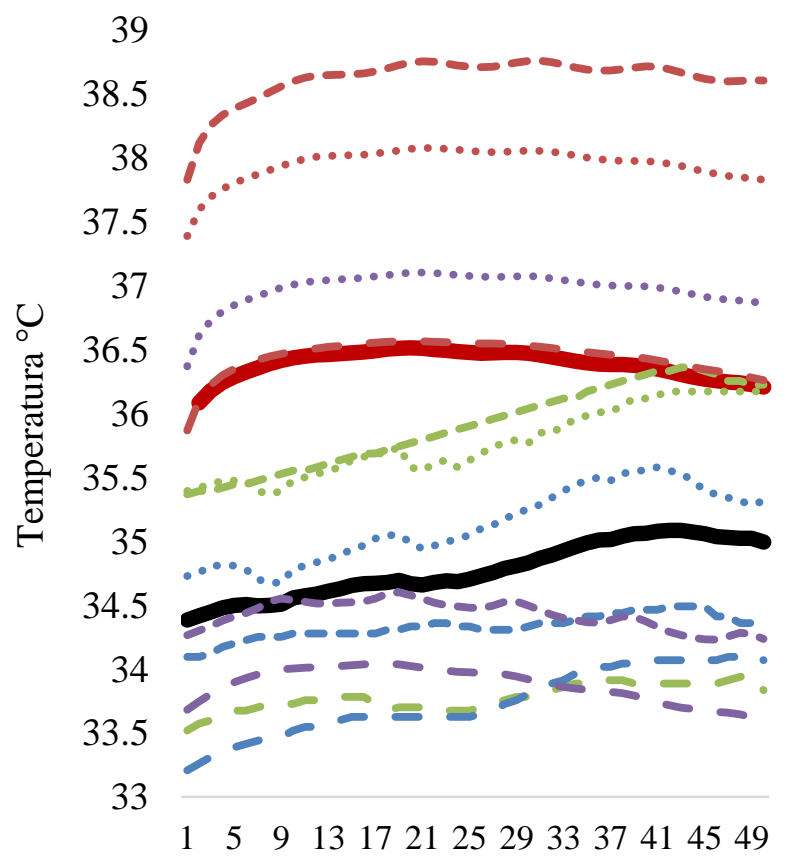

Datos en posición Y (Largo del cañón)

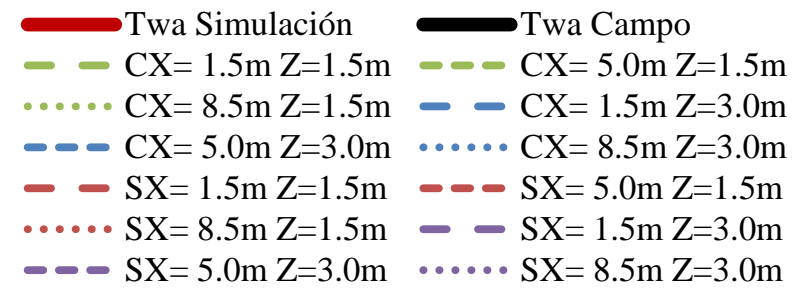

Gráfica 1 Comportamiento del promedio de la temperatura del aire $\left(\mathrm{T}_{\mathrm{WA}}\right)$ a diferentes alturas $(\mathrm{Z}) \mathrm{y}$ posiciones a lo ancho de la calle $(\mathrm{X})$. La $\mathrm{C}$ es para mediciones en campo y la $\mathrm{S}$ para valores obtenidos por simulación

Fuente: Elaboración Propia
Los valores que más difieren son los ubicados a 1.50 metros de altura entre campo y simulación, con variación de hasta $2^{\circ} \mathrm{C}$, sin embargo, las líneas con los datos en la posición $\mathrm{Z}=3.0$ metros son variaciones de menos de $0.20^{\circ} \mathrm{C}$.

\section{Análisis y resultados}

En total se realizaron 36 simulaciones, todas con constante de velocidad de viento de $1.2 \mathrm{~m} / \mathrm{s}$, con dos orientaciones de Este-Oeste y Norte-Sur, tres variables de número de coches (cero, diez y veinte), y seis tipos de relaciones de aspecto $(\mathrm{H} / \mathrm{W}=$ altura/ancho).

Tanto la simulación, como las mediciones de campo fueron realizadas a las 13 horas, por lo que la fachada Este y el pavimento han llegado a su máxima exposición solar y capacidad térmica en el cenit. En la Tabla 3 se muestran las combinaciones y los resultados de la temperatura promedio del aire interna del cañón.

Los valores más bajos se encuentran ubicados en los cañones Este-Oeste, indistintamente del ancho que tengan, variando ente los 30 a los $32^{\circ} \mathrm{C}$. Las calles orientadas al Norte-Sur, sus variaciones son más drásticas y oscilan entre los 33 a los $38^{\circ} \mathrm{C}$, superando a los anteriores con casi $6^{\circ} \mathrm{C}$.

\begin{tabular}{|c|c|c|c|}
\hline Casos de & 0 coches & 10 coches & 20 coches \\
\hline & $\begin{array}{l}T_{\text {WA }} \\
{ }^{\circ} \mathrm{C}\end{array}$ & $\begin{array}{l}\mathrm{T}_{\mathrm{WA}} \\
{ }^{\circ} \mathrm{C}\end{array}$ & $\begin{array}{l}T_{\text {WA }} \\
{ }^{\circ} \mathrm{C}\end{array}$ \\
\hline $1.2 \mathrm{~m} / \mathrm{s}$ E-O 10:05 & 30.55 & 31.20 & 31.70 \\
\hline $1.2 \mathrm{~m} / \mathrm{s} \mathrm{E}-\mathrm{O} 10: 10$ & 30.61 & 31.08 & 31.53 \\
\hline $1.2 \mathrm{~m} / \mathrm{s} \mathrm{E}-\mathrm{O} 10: 20$ & 30.57 & 31.71 & 32.25 \\
\hline Promedio E-O W=10 & 30.58 & 31.33 & 31.82 \\
\hline $1.2 \mathrm{~m} / \mathrm{s}$ E-O 20:05 & 30.51 & 31.05 & 32.90 \\
\hline $1.2 n$ & 30.58 & 30.99 & 31.57 \\
\hline $1.2 \mathrm{~m} / \mathrm{s} \mathrm{E}-\mathrm{O} 20: 20$ & 30.64 & 31.03 & 31.51 \\
\hline Promedio E-O W=20 & 30.58 & 31.02 & 32.00 \\
\hline $1.2 \mathrm{~m} / \mathrm{s} \mathrm{N}-\mathrm{S} 10: 05$ & 34.85 & 36.37 & 37.61 \\
\hline $1.2 \mathrm{~m} / \mathrm{s} \mathrm{N}-\mathrm{S} 10: 10$ & 35.12 & 36.69 & 38.15 \\
\hline $1.2 \mathrm{~m} / \mathrm{s} \mathrm{N}-\mathrm{S} 10: 20$ & 34.51 & 37.09 & 38.28 \\
\hline Promedio N-S W=10 & 34.83 & 36.72 & 38.01 \\
\hline $1.2 \mathrm{~m} / \mathrm{s} \mathrm{N}-\mathrm{S} 20: 05$ & 33.76 & 34.88 & 35.96 \\
\hline $1.2 \mathrm{~m} / \mathrm{s} \mathrm{N}-\mathrm{S} 20: 10$ & 33.78 & 34.74 & 35.82 \\
\hline $1.2 \mathrm{~m} /$ & 33.73 & 34.50 & 35.47 \\
\hline Promedio N-S W=20 & 33.75 & 34.70 & 35.75 \\
\hline
\end{tabular}

Tabla 3 Los 36 casos de simulación, realizados en ANSyS Fuente: Elaboración Propia 
La Figura 7, muestra el comportamiento del viento en el centro del cañón, donde se observa como recoge el calor de los primeros coches y se disipa a lo largo de la calle.

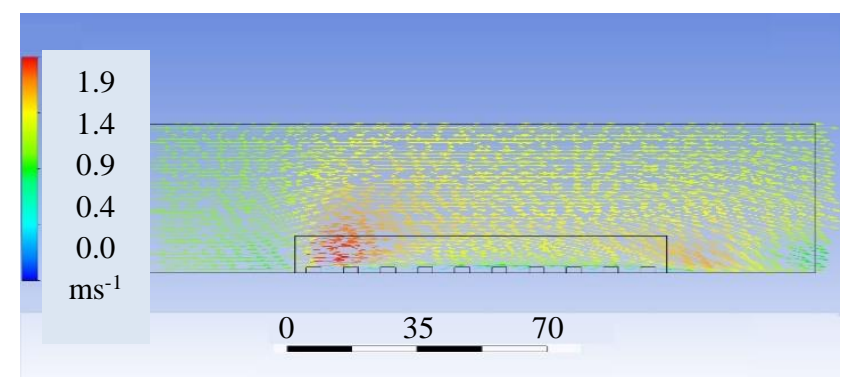

Figura 7 Corte longitudinal de la velocidad del viento, en un cañón de 10 unidades, con orientación Norte-Sur, relación aspecto de $\mathrm{H} / \mathrm{W}=10 / 20$ metros

Fuente: Elaboración Propia

El perfil converge a la velocidad del viento convenida en el programa conforme asciende sobre los edificios, pero se aprecia un incremento de velocidad al inicio del cañón provocando vórtices y aumento en la velocidad; posteriormente hay una desaceleración y por lo tanto disminución del viento en su capacidad de convección.

Implicando que las mayores temperaturas se encuentren localizadas en la parte posterior del cañón donde el viento pierde su capacidad portante y disipante de calor.

La Figura 8, es el corte de la simulación con relación de aspecto $\mathrm{H} / \mathrm{W}=10 / 10=1$ y con orientación Norte-Sur, ubicado a los 50 metros, exactamente a la mitad de la calle.

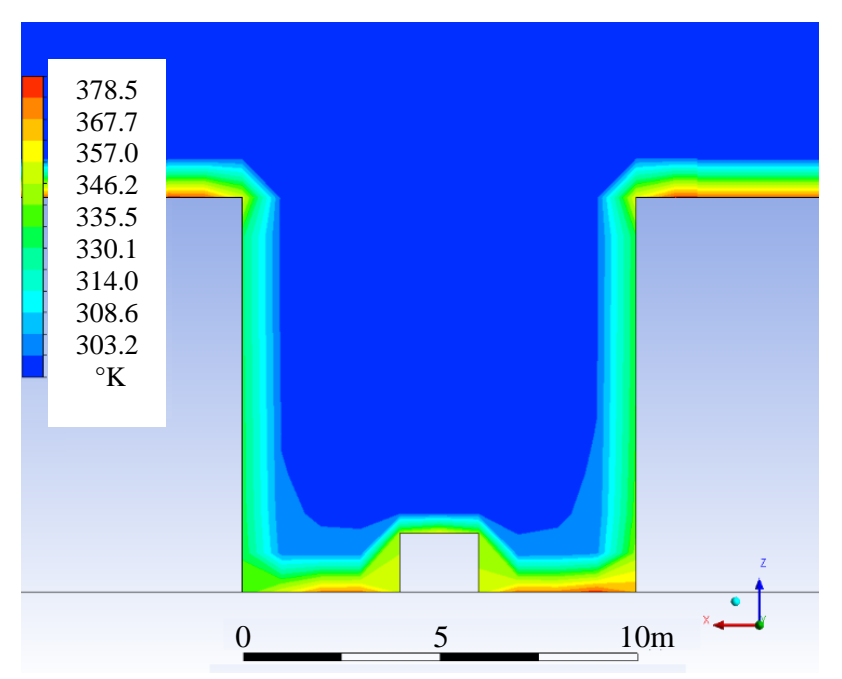

Figura 8 Corte transversal de la temperatura interna en un cañón de 10 unidades, con orientación Norte-Sur, relación aspecto de $\mathrm{H} / \mathrm{W}=10 / 10$ metros

Fuente: Elaboración Propia
En el corte, las temperaturas mayores son localizadas en la azotea y en el pavimento, sin embargo, las fachadas presentan un incremento considerable de $55^{\circ} \mathrm{C}$ que afectan el comportamiento energético de los edificios, y la parte inferior, donde se encuentra el transeúnte está altamente afecta hasta la altura de 1 metros con temperaturas similares.

\section{Análisis grafico por altura $(Z)$ y posición en ancho de la calle $(X)$}

Las presentes gráficas de superficie representan en el eje de las abscisas: los promedios de temperatura por cada línea en la simulación, en $\mathrm{Z}(1.5,3.0$ y 4.5 metros) y por cada combinación que esta tenga en el eje de la X $(1.5,5.0$ y 8.5 metros), de acuerdo con las diferentes configuraciones de altura y ancho (eje de las ordenadas).

Las Gráficas 2, 3 y 4, son los cañones orientados al Este-Oeste, con una diferencia de 0,10 y 20 coches en su interior, como observamos sus comportamientos son muy estables junto a las fachadas (posición $\mathrm{X}=1.5 \mathrm{y}$ 8.5 en cañones de 10 metros de ancho) sin importar la altura en el eje Z, no rebasan los $32^{\circ} \mathrm{C}$.

Sin embargo, el comportamiento en los centros $(x=5 \quad 0 \quad x=10 m)$ presenta variaciones drásticas, en especial a la altura de 1.5 metros, con diferencias marcadas hasta de $38^{\circ} \mathrm{C}$.

El siguiente set de Gráficas 5,6 y 7 son cañones orientados al igual que las anteriores, Este-Oeste, pero con un ancho de 20 metros, al igual que los anteriores el comportamiento en las posiciones de $\mathrm{X}(1.5$ y $18.5 \mathrm{~m})$ son estables, presentando un grado menos que las gráficas 2 , $3, \mathrm{y} 4,31^{\circ} \mathrm{C}$.

El comportamiento más inestable es a la altura de $1.5 \mathrm{~m}$ en el centro, con valores altos de $39^{\circ} \mathrm{C}$. 


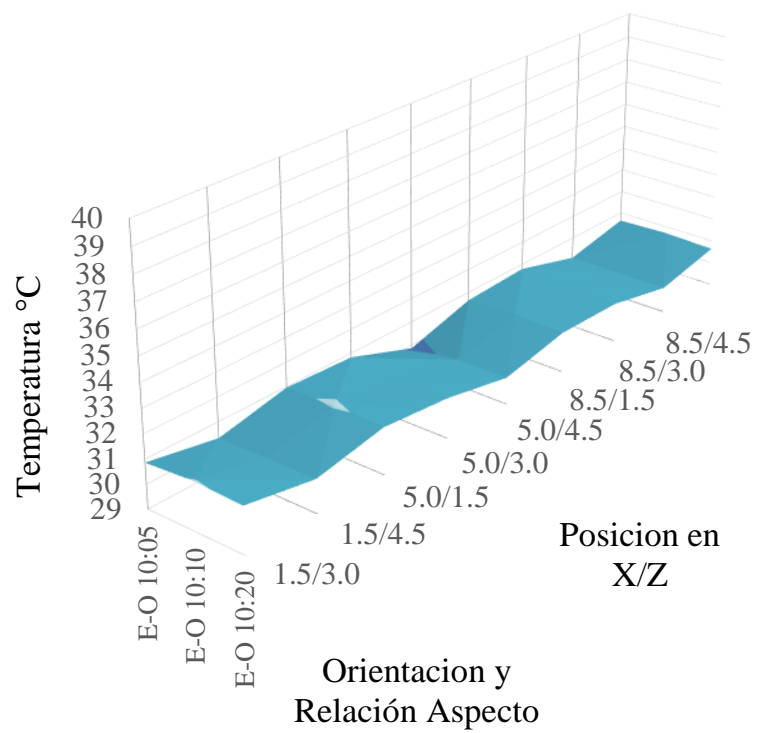

Gráfica 2 Comportamiento del promedio de la temperatura del aire $\left(\mathrm{T}_{\mathrm{WA}}\right)$, a diferentes alturas $(\mathrm{Z})$ y posición a lo ancho de la calle $(\mathrm{X})$, sin ningún vehículo y orientados al ESTE-OESTE (fachadas hacia el Norte y Sur). son los datos de un cañón con relación de aspecto de $\mathrm{H} / \mathrm{W}=5 / 10=0.5 ; 10 / 10=1$ y $20 / 10=2$

Fuente: Elaboración Propia

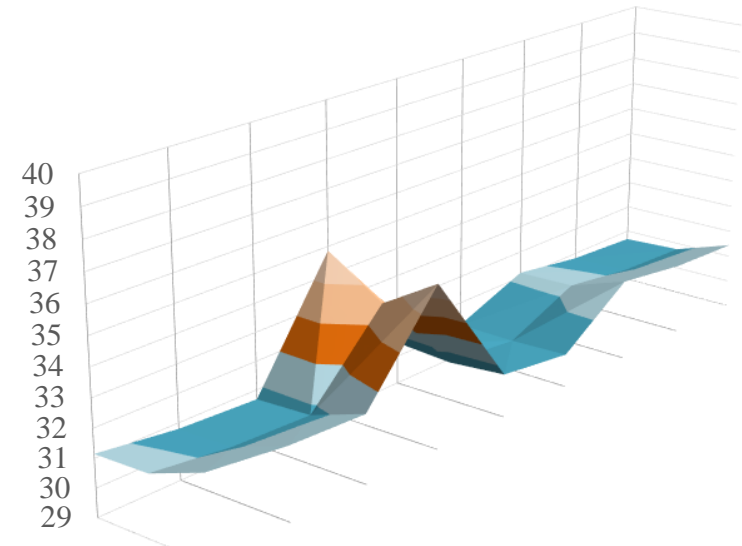

Gráfica 3 Mismas constantes que la gráfica 2, pero con 10 vehículos en su interior Fuente: Creación Propia

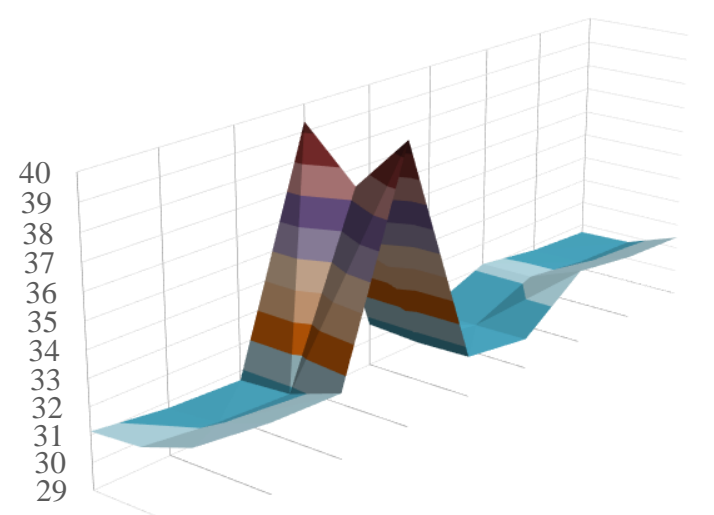

Gráfica 4. Mismas constantes que la gráfi5 ca 2, pero con 20 vehículos en su interior Fuente: Elaboración Propia

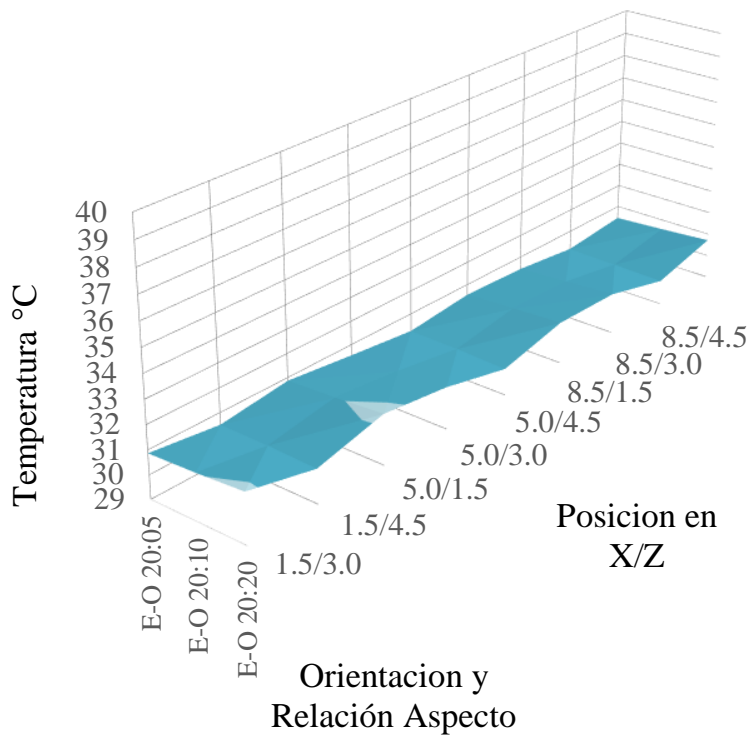

Gráfica 5 Comportamiento del promedio de la temperatura del aire $\left(\mathrm{T}_{\mathrm{WA}}\right)$, a diferentes alturas $(\mathrm{Z})$ y posición a lo ancho de la calle $(\mathrm{X})$, sin ningún vehículo y orientados al ESTE-OESTE (fachadas hacia el Norte y Sur). son los datos de un cañón con relación de aspecto de $\mathrm{H} / \mathrm{W}=5 / 20=0.25 ; 10 / 20=0.5$ y $20 / 20=1$

Fuente: Elaboración Propia

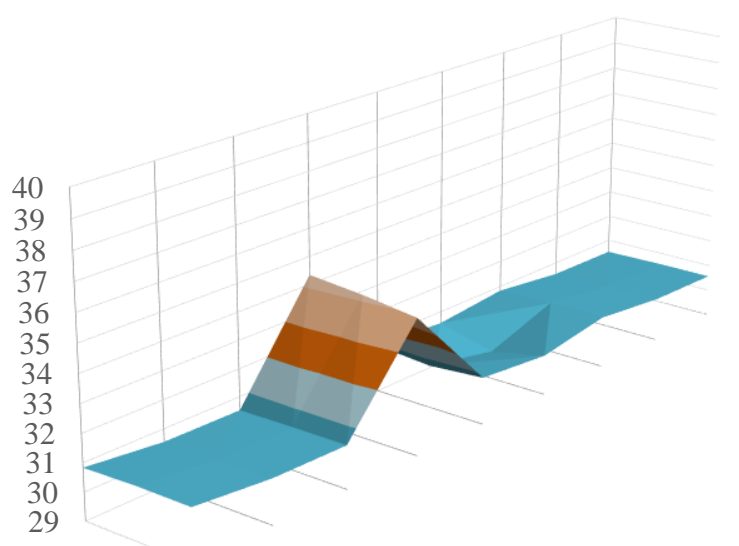

Gráfica 6 Mismas constantes que la gráfica 5, pero con 10 vehículos en su interior

Fuente: Elaboración Propia

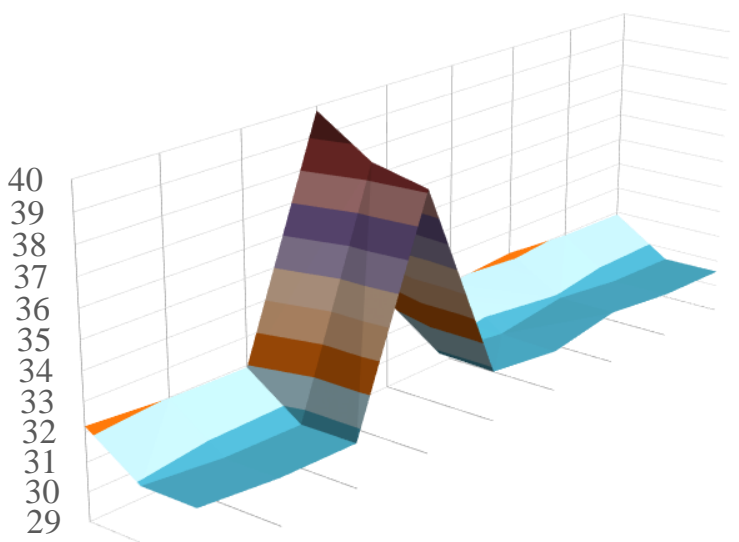

Gráfica 7 Mismas constantes que la gráfica 5, pero con 10 vehículos en su interior

Fuente: Elaboración Propia 


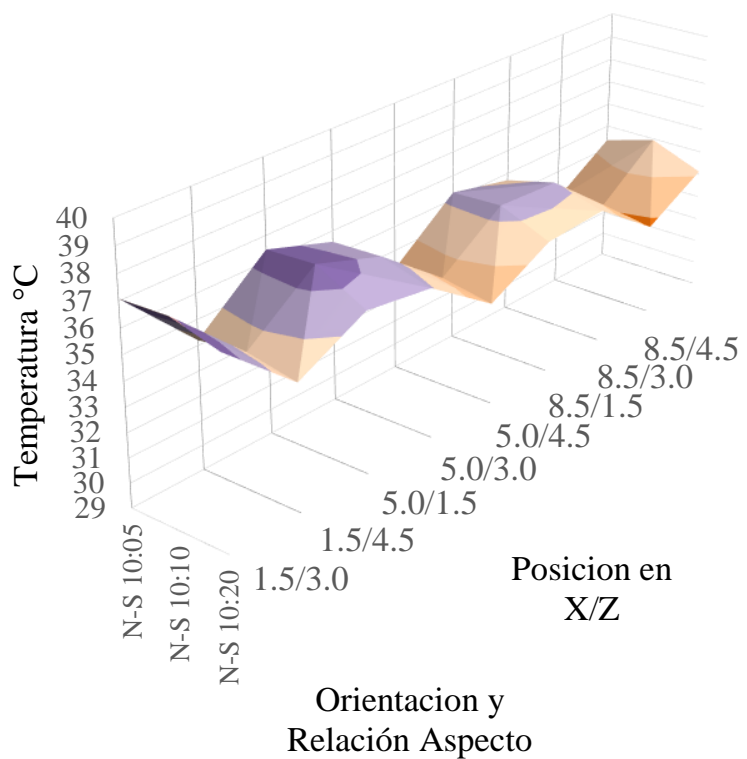

Gráfica 8 Comportamiento del promedio de la temperatura del aire ( $\mathrm{T}_{\mathrm{WA}}$ ), a diferentes alturas $(\mathrm{Z})$ y posición a lo ancho de la calle $(\mathrm{X})$, sin ningún vehículo y orientados al NORTE-SUR (fachadas hacia el Este y Oeste). son los datos de un cañón con relación de aspecto de $\mathrm{H} / \mathrm{W}=5 / 10=0.5 ; 10 / 10=1$ y $20 / 10=2$

Fuente: Elaboración Propia

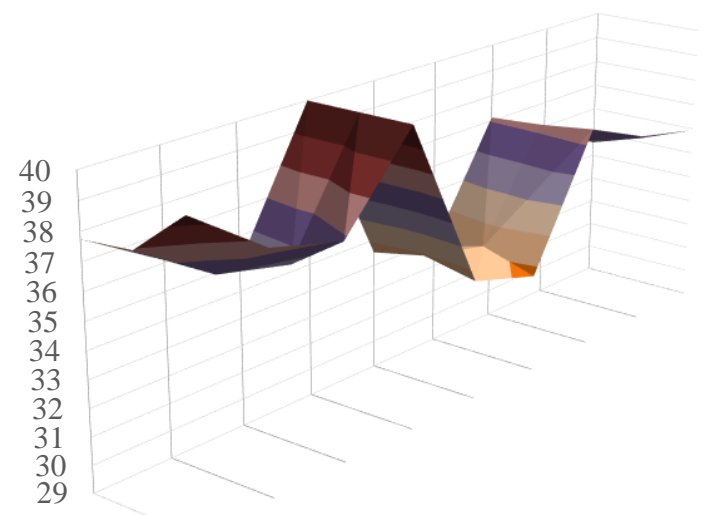

Gráfica 9. Mismas constantes que la gráfica 8, pero con 10 vehículos en su interior

Fuente: Elaboración Propia

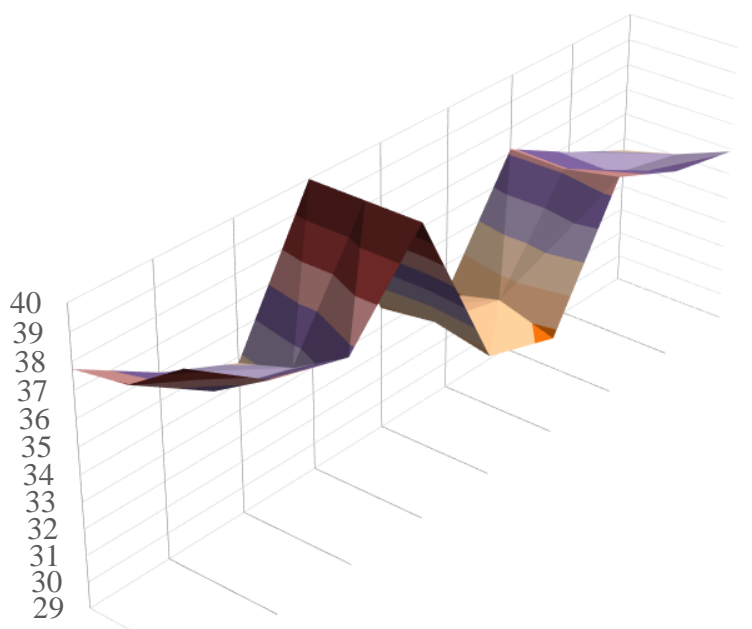

Gráfica 10. Mismas constantes que la gráfica 8, pero con 20 vehículos en su interior Fuente: Elaboración Propia

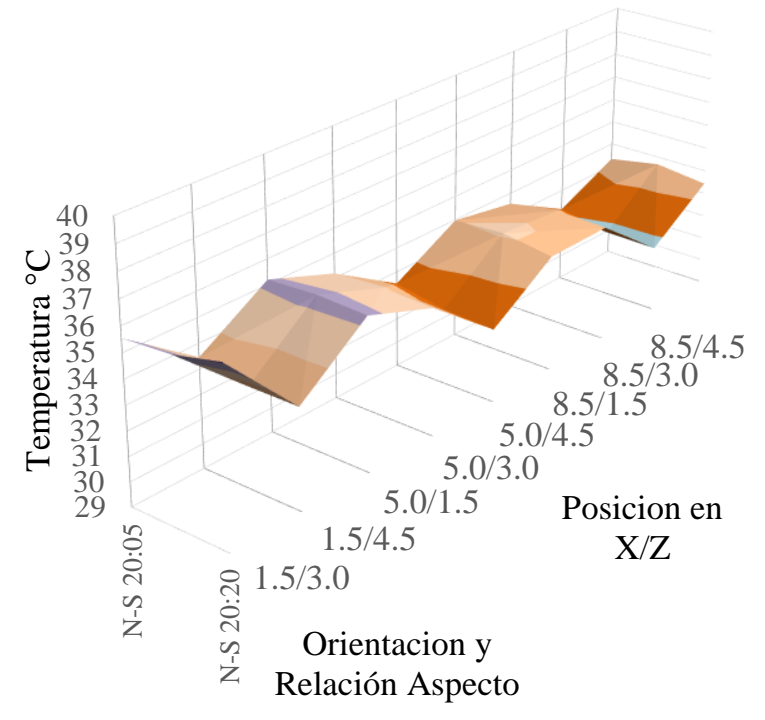

Gráfica 11 Comportamiento del promedio de la temperatura del aire ( $\mathrm{T}_{\mathrm{WA}}$ ), a diferentes alturas ( $\left.\mathrm{Z}\right)$ y posición a lo ancho de la calle $(\mathrm{X})$, sin ningún vehículo y orientados al NORTE-SUR (fachadas hacia el Este y Oeste). son los datos de un cañón con relación de aspecto de $\mathrm{H} / \mathrm{W}=5 / 20=0.25 ; 10 / 20=0.5$ y $20 / 20=1$.

Fuente: Elaboración propia

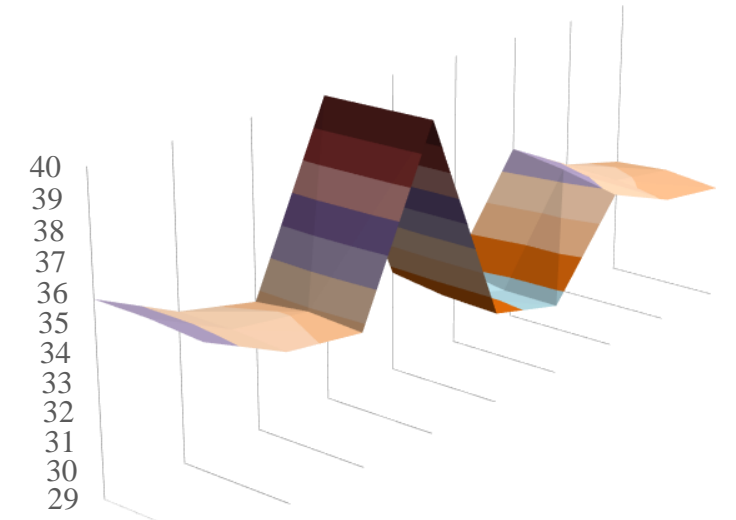

Gráfica 12 Mismas constantes que la gráfica 11, pero con 10 vehículos en su interior Fuente: Elaboración Propia

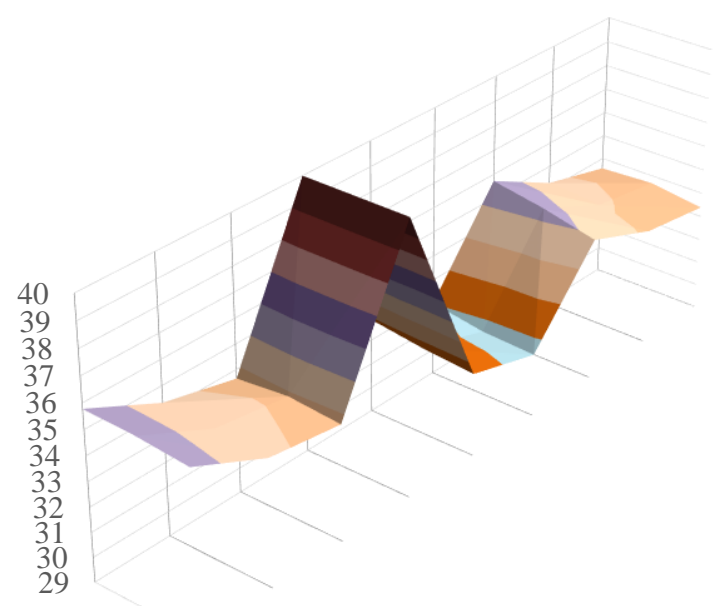

Gráfica 13 Mismas constantes que la gráfica 11, pero con 20 vehículos en su interior Fuente: Elaboración Propia

GRAJEDA-ROSADO, Ruth María, ALONSO-GUZMAN, Elia Mercedes, ESPARZA-LOPEZ, Carlos Javier y ESCOBAR-DEL POZO, Carlos. Simulación del comportamiento térmico en exteriores urbanos correlacionando las variables de calor antropogénico vehicular y orientación. Revista de Simulación y Laboratorio. 2019. 


$\begin{array}{lll}\square 29.00-30.00 & \square 30.00-31.00 & \square 31.00-32.00 \\ \square 32.00-33.00 & \square 33.00-34.00 & -34.00-35.00 \\ \square 35.00-36.00 & \square 36.00-37.00 & \square 37.00-38.00 \\ \square 38.00-39.00 & \square 39.00-40.00 & \end{array}$

Las Gráficas 8, 9 y 10 son muy diferentes y menos estables que las anteriores, debido a su orientación (Este-Oeste), en este caso la temperatura promedio oscila entre los $34 \mathrm{a} 35^{\circ} \mathrm{C}$, presentando mayor temperatura que los orientados al Norte-Sur.

En las posiciones $\mathrm{X}$, es decir junto a las fachadas, los valores pueden llegar hasta los de 36 a $37^{\circ} \mathrm{C}$, y los centros ( $\mathrm{X}=1.5$ metros) a una altura de 1.5 metros, la temperatura con 10 y 20 coches se quedan con $39^{\circ} \mathrm{C}$.

Es importante resaltar que los datos en la posición del centro, a una altura de $4.5 \mathrm{~m}$, son las más bajas con $32^{\circ} \mathrm{C}$, reafirmando lo que se observa en la Figura 8, donde el centro del cañón presenta su temperatura menor.

Las Gráficas 11, 12 y 13, con orientación a Norte-Sur y con ancho de 20 metros, presentan un comportamiento parecido a las anteriores, pero sus valores casi bajan $2^{\circ} \mathrm{C}$ en todos los demás puntos de análisis.

Junto a fachadas se pueden observar valores de $34^{\circ} \mathrm{C}$ a una altura de 4.5 metros, ascendiendo a $35^{\circ} \mathrm{C}$ a 1.5 metros. Los valores en los centros del cañón llegan a $39^{\circ} \mathrm{C}$ pero a una altura de 4.5 , descienden a $32^{\circ} \mathrm{C}$, por lo que la graficas con 10 y 20 coches presentan comportamientos más pronunciados.

En la Gráfica 11, es decir sin coches los valores son más uniformes que su contraparte la Gráfica 8, teniendo valores promedio de $34^{\circ} \mathrm{C}$; en comparación con los $37^{\circ} \mathrm{C}$ que demuestra la gráfica con una relación de aspecto de $1, \mathrm{H} / \mathrm{W}=$ $10 / 10$ metros.

Las gráficas anteriores demostraron el comportamiento de los cañones, analizando sus temperaturas promedio por posición de altura (Z) y ubicación en el ancho de la calle (X).

\section{Análisis por número de coches}

Mediante la Tabla 4, se analizan los porcentajes de aumentos por incremento de número de coches, la comparación se hace tomando como casos bases, los cañones con sus diferentes configuraciones, pero sin autos. Las diferencias son expresadas en porcentaje, donde podemos afirmar que:

a. Al agregar 10 unidades en el cañón el incremento en promedio en los orientados Este-Oeste es de $2 \%$ y con 20 unidades, es proporcional con un $4 \%$.

b. Los casos menos favorecidos son los orientados al Norte-Sur y presentan un promedio de $4 \%$ más por 10 unidades y $7.5 \%$ por 20 unidades.

c. Los cañones con 20 metros de ancho presentan los menores incrementos en relación con los de 10 metros, con base a esta variable.

d. El caso más crítico lo obtiene el cañón de 10 metros de ancho con 20 metros de altura y 20 coches, con $11 \%$ de incremento, pudiendo ser una confirmación de la investigación en una escala menor como una manzana, Ichionse (1999), donde lo asocia con la altura de los edificios (número de pisos).

e. Los casos más favorecidos son los cañones de 20 metros de ancho, con una orientación Este-Oeste, obteniendo valores en promedio de $1.5 \%$ más que la base comparación, es decir 0 unidades.

f. La comparación entre las relaciones de aspecto, podemos ver que los orientados Este-Oeste, sus diferencias no son tan altas, variando entre 2 a $3 \%$ más, sin embargo, los orientados al Norte-Sur, pueden casi duplicar su valor. 


\begin{tabular}{|c|c|c|c|}
\hline Caso & $\begin{array}{l}0 \text { unidades } \\
\text { Valor }^{\circ} \mathrm{C}\end{array}$ & $\begin{array}{l}10 \text { coches } \\
\%\end{array}$ & $\begin{array}{l}20 \text { coche } \\
\%\end{array}$ \\
\hline $1.2 \mathrm{~m} / \mathrm{s} \mathrm{E}-\mathrm{O} 10: 05$ & 30.55 & 2.11 & 3.75 \\
\hline $1.2 \mathrm{~m} / \mathrm{s} \mathrm{E}-\mathrm{O} 10: 10$ & 30.61 & 1.52 & 2.99 \\
\hline $1.2 \mathrm{~m} / \mathrm{s} \mathrm{E}-\mathrm{O} 10: 20$ & 30.57 & 3.73 & 5.47 \\
\hline $1.2 \mathrm{~m} / \mathrm{s}$ E-O $20: 05$ & 30.51 & 1.75 & 3.84 \\
\hline $1.2 \mathrm{~m} / \mathrm{s}$ E-O $20: 10$ & 30.58 & 1.35 & 3.25 \\
\hline $1.2 \mathrm{~m} / \mathrm{s} \mathrm{E}-\mathrm{O} 20: 20$ & 30.64 & 1.25 & 2.84 \\
\hline $1.2 \mathrm{~m} / \mathrm{s} \mathrm{N}-\mathrm{S} 10: 05$ & 34.85 & 4.36 & 7.91 \\
\hline $1.2 \mathrm{~m} / \mathrm{s} \mathrm{N}-\mathrm{S} 10: 10$ & 35.12 & 4.45 & 8.62 \\
\hline $1.2 \mathrm{~m} / \mathrm{s} \mathrm{N}-\mathrm{S} 10: 20$ & 34.51 & 7.48 & 10.94 \\
\hline $1.2 \mathrm{~m} / \mathrm{s} \mathrm{N}-\mathrm{S} 20: 05$ & 33.76 & 3.30 & 6.51 \\
\hline $1.2 \mathrm{~m} / \mathrm{s} \mathrm{N}-\mathrm{S} 20: 10$ & 33.78 & 2.84 & 6.07 \\
\hline $1.2 \mathrm{~m} / \mathrm{s} \mathrm{N}-\mathrm{S} 20: 20$ & 33.73 & 2.29 & 5.17 \\
\hline Orientación E-O & Promedio & 1.95 & 4.36 \\
\hline Orientación N-S & Promedio & 4.12 & 7.54 \\
\hline
\end{tabular}

Tabla 4 Comparación de datos en relación con el número de autos

Fuente: Elaboración Propia

\section{Análisis por orientación}

Los valores determinados con esta variable son los más altos y al mismo tiempo los más uniformes. Tomando como base los cañones orientados al Este-Oeste, se comparan con los orientados al Norte-Sur.

Con la Tabla 5, se realizan las siguientes conclusiones:

a. Los cañones con 10 metros de ancho (W), en promedio muestran un incremento progresivo, de 3\% más; por cada 10 unidades que ingresan en el cañón.

b. Los cañones de 20 metros de ancho no parecen variar mucho, presentando un $10 \%$ en promedio para los 0 coches y $12 \%$ por las 10 y 20 unidades que se encuentran generando calor gracias al motor de combustión y la radiación difusa de la carrocería.

c. Si se examina los porcentajes entre los cañones de 10 y 20 metros de ancho, se observa un fuerte incremento con los orientados Este-Oeste

d. El caso más crítico lo presenta el cañón Norte-Sur con una relación de aspecto (RA) de $1(\mathrm{H} / \mathrm{W}=10 / 10 \mathrm{~m})$. e. El valor menor, lo ostenta el cañón con orientación $\mathrm{N}-\mathrm{S}$, con una RA= $0.25(\mathrm{H} / \mathrm{W}=$ $5 / 20 \mathrm{~m}$ ) con $9 \%$ más, le siguen los dos casos de $10 \%$ que son los cañones con 20 metros de ancho, los cuales son el de $\mathrm{RA}=0.5 \quad(\mathrm{H} / \mathrm{W}=10 / 20) \quad \mathrm{y} \quad$ el $\quad \mathrm{RA}=1$ $(\mathrm{H} / \mathrm{W}=20 / 20)$.

f. La penúltima conclusión, permite concluir que, aunque el caso más crítico tiene un $\mathrm{RA}=1(10 / 10)$, la misma $\mathrm{RA}=1(20 / 20)$ sea un caso menor, confirmando que la apertura en la calle permite un mayor flujo de movimiento de aire, y una ventana térmica en promedio general, esto no implica que lo valores sean menoras a los costados de las fachadas.

\begin{tabular}{|c|c|c|c|}
\hline $\begin{array}{c}\text { Caso } \\
\text { Valor inicial }^{\circ} \mathrm{C}\end{array}$ & $\begin{array}{c}0 \\
\text { unidades }\end{array}$ & $\begin{array}{c}10 \\
\text { coches }\end{array}$ & $\begin{array}{c}20 \\
\text { coches }\end{array}$ \\
\hline $1.2 \mathrm{~m} / \mathrm{s}$ E-O 10:05 & 30.55 & 31.20 & 31.70 \\
\hline $1.2 \mathrm{~m} / \mathrm{s} \mathrm{N}-\mathrm{S} 10: 05$ & $14 \%$ & $17 \%$ & $19 \%$ \\
\hline $1.2 \mathrm{~m} / \mathrm{s}$ E-O $10: 10$ & 30.61 & 31.08 & 31.53 \\
\hline $1.2 \mathrm{~m} / \mathrm{s}$ N-S $10: 10$ & $15 \%$ & $18 \%$ & $21 \%$ \\
\hline $1.2 \mathrm{~m} / \mathrm{s}$ E-O $10: 20$ & 30.57 & 31.71 & 32.25 \\
\hline $1.2 \mathrm{~m} / \mathrm{s} \mathrm{N}-\mathrm{S} 10: 20$ & $13 \%$ & $17 \%$ & $19 \%$ \\
\hline $\begin{array}{l}\text { Promedio } \\
\mathrm{W}=10 \mathrm{~m}\end{array}$ & $14 \%$ & $17 \%$ & $19 \%$ \\
\hline $1.2 \mathrm{~m} / \mathrm{s}$ E-O $20: 05$ & 30.51 & 31.05 & 32.90 \\
\hline $1.2 \mathrm{~m} / \mathrm{s}$ N-S 20:05 & $11 \%$ & $12 \%$ & $9 \%$ \\
\hline $1.2 \mathrm{~m} / \mathrm{s}$ E-O $20: 10$ & 30.58 & 31.00 & 31.57 \\
\hline $1.2 \mathrm{~m} / \mathrm{s}$ N-S $20: 10$ & $10 \%$ & $12 \%$ & $13 \%$ \\
\hline $1.2 \mathrm{~m} / \mathrm{s}$ E-O $20: 20$ & 30.64 & 31.03 & 31.51 \\
\hline $1.2 \mathrm{~m} / \mathrm{s} \mathrm{N}-\mathrm{S} 20: 20$ & $10 \%$ & $11 \%$ & $13 \%$ \\
\hline $\begin{array}{l}\text { Promedio } \\
\text { W=20m }\end{array}$ & $10 \%$ & $12 \%$ & $12 \%$ \\
\hline
\end{tabular}

Tabla 5 Base de datos para la comparación de las simulaciones con cañones orientados al Este-Oeste y su porcentaje de aumento con los orientados al Norte-Sur Fuente: Elaboración propia

\section{Análisis por relación de aspecto}

La última variable de análisis son las relaciones de aspecto (RA), en los 36 casos de estudio. En este caso, se toma como base los cañones con menor altura, es decir $\mathrm{RA}=0.5(\mathrm{H} / \mathrm{W}=5 / 10)$ y $\mathrm{RA}=0.25(\mathrm{H} / \mathrm{W}=5 / 20 \mathrm{~m})$.

Los resultados se vuelven más disimiles y difíciles de suponer reglas que los anteriores como se observa en la Tabla 6 , los valores están dado en porcentaje y los positivos significan aumento y los negativos disminución de calor en la temperatura promedio con relación al caso base. 
a. Los casos más favorables para disminuir la temperatura son encontrados en los cañones E-O con veinte unidades de autos y un ancho de 20metros, podemos decir que, aunque en estos casos se aumenta más la temperatura con las otras variables, el ancho de la calle favorece a disipar el calor generado por los autos, gracias a la modificación de la geometría de la calle.

b. El caso con el valor más alto, lo posee el cañón orientado al Norte-Sur con una relación de aspecto de $2(\mathrm{H} / \mathrm{W}=20 / 10 \mathrm{~m})$, donde por su altura alberga más el calor de los autos, siendo de $1.8 \%$ el incremento.

c. En general los cañones con N-S y de 10 metros de ancho, son los que menos disipan el flujo de calor.

d. Los que presenten menos alteración, son los cañones con 20 metros de ancho, orientación $\mathrm{N}-\mathrm{S}$, sin coches, donde su promedio es general es de $-0.03 \%$.

\begin{tabular}{|c|c|c|c|}
\hline $\begin{array}{c}\text { Caso } \\
\text { Valor inicial }{ }^{\circ} \mathbf{C}\end{array}$ & $\begin{array}{c}0 \\
\text { unidades }\end{array}$ & $\begin{array}{c}10 \\
\text { coches }\end{array}$ & $\begin{array}{c}20 \\
\text { coches }\end{array}$ \\
\hline $1.2 \mathrm{~m} / \mathrm{s}$ E-O 10:05 & 30.55 & 31.20 & 31.70 \\
\hline $1.2 \mathrm{~m} / \mathrm{s}$ E-O $10: 10$ & $0.20 \%$ & $-0.37 \%$ & $0.54 \%$ \\
\hline $1.2 \mathrm{~m} / \mathrm{s}$ E-O $10: 20$ & $0.06 \%$ & $1.66 \%$ & $1.73 \%$ \\
\hline $\begin{array}{ll}\text { Promedio } & \text { E-O, } \\
\mathrm{W}=10 & \\
\end{array}$ & $0.13 \%$ & $0.64 \%$ & $0.59 \%$ \\
\hline $1.2 \mathrm{~m} / \mathrm{s}$ E-O $20: 05$ & 30.51 & 31.05 & 32.90 \\
\hline $1.2 \mathrm{~m} / \mathrm{s}$ E-O $20: 10$ & $0.21 \%$ & $-0.18 \%$ & $4.05 \%$ \\
\hline $1.2 \mathrm{~m} / \mathrm{s}$ E-O $20: 20$ & $0.43 \%$ & $-0.06 \%$ & $4.23 \%$ \\
\hline $\begin{array}{l}\text { Promedio E-O, } \\
\mathrm{W}=20\end{array}$ & $0.32 \%$ & $-0.12 \%$ & $4.14 \%$ \\
\hline $1.2 \mathrm{~m} / \mathrm{s} \mathrm{N}-\mathrm{S} 10: 05$ & 34.85 & 36.37 & 37.61 \\
\hline $1.2 \mathrm{~m} / \mathrm{s} \mathrm{N}-\mathrm{S} 10: 10$ & $0.79 \%$ & $0.88 \%$ & $1.45 \%$ \\
\hline $1.2 \mathrm{~m} / \mathrm{s} \mathrm{N}-\mathrm{S} 10: 20$ & $-0.98 \%$ & $1.99 \%$ & $1.80 \%$ \\
\hline $\begin{array}{l}\text { Promedio N-S, } \\
\mathrm{W}=10\end{array}$ & $-0.10 \%$ & $1.43 \%$ & $1.63 \%$ \\
\hline $1.2 \mathrm{~m} / \mathrm{s} \mathrm{N}-\mathrm{S} 20: 05$ & 33.76 & 34.88 & 35.96 \\
\hline $1.2 \mathrm{~m} / \mathrm{s} \mathrm{N}-\mathrm{S} 20: 10$ & $0.04 \%$ & $-0.40 \%$ & $0.37 \%$ \\
\hline $1.2 \mathrm{~m} / \mathrm{s} \mathrm{N}-\mathrm{S} 20: 20$ & $-0.11 \%$ & $-1.08 \%$ & $1.35 \%$ \\
\hline $\begin{array}{l}\text { Promedio N-S, } \\
\mathrm{W}=20\end{array}$ & $-0.03 \%$ & $-0.74 \%$ & $0.86 \%$ \\
\hline
\end{tabular}

Tabla 6 Base de datos para la comparación de las simulaciones en relaciones de aspecto, la base dada en ${ }^{\circ} \mathrm{C}$ son los cañones que contiene edificios con altura de 5metros

Fuente: Elaboración Propia

\section{Agradecimientos}

Al Centro de Investigaciones en Micro y Nanotecnología de la Universidad Veracruzana por el financiamiento de los equipos y sensores, en especial por el dron, así como al Heroico Ayuntamiento de Veracruz por los servicios de logística vial y permisos de vuelo dentro de una zona urbana.

\section{Conclusiones}

Como la literatura lo ha mencionado, el calor antropogénico vehicular presente muchas variables para su análisis y cuantiosas consideraciones para la recolección de datos en campo.

La presente investigación se basa en la realización de 36 simulaciones en CFD de un ambiente urbano idealizado, para entender un factor de formación de la ICU, que es $\mathrm{Q}_{\mathrm{FV}}$, al lograr correlacionar esta variable con la orientación, relaciones de aspecto y número de coches que transitan en una calle.

Sobre los datos tomados en campo para la validación de las presentes simulaciones (Grajeda et al, 2018), cabe apuntar, que el documento determinó que el movimiento de los automóviles con velocidades constantes generaba corrientes de aire que disipaban el calor hacia la acera de la calle, exponiendo que el tráfico estacionario fomenta mayor calor sensible.

Es por lo tanto que los resultados se manejan como promedios generales; un objetivo más adelante es diseñar una metodología para analizar cómo afecta la velocidad del auto, al incremento o decremento del calor dentro de una calle, es decir, definir valores más realistas para QFV.

La mayor conclusión de los análisis de datos es que la orientación Norte-Sur del cañón (fachadas hacia Este-Oeste) son las que representan mayor elevación en el perfil térmico, debido a que el recorrido solar atraviesa de forma perpendicular; ocasionando que tanto el área horizontal (calle) y la fachada orientada hacia el Este, suman el doble de área calentada por la radiación solar, incrementando, por lo tanto, el valor de afectación por los autos con la radiación difusa. 
Por lo que algunas técnicas pasivas deben ser consideras, en especial para los cañones N-S $\mathrm{y}$ configuraciones angostas. Estas técnicas pueden ser; determinar su aforo vehicular; generar un flujo constante o movilidad vehicular y elementos constructivos que ayuden en la disipación del calor y aumento de la turbulencia del viento.

Al igual que el trabajo de Llaguno et al. (2017), la geometría de las características arquitectónicas de los edificios puede ser beneficiosa aumentado la intensidad de la turbulencia del flujo entrante y saliente de la calle, donde aseguran que los techos de formas complejas (redondos o inclinados) dan como resultado una mayor intensidad de turbulencia y mezcla del aire, en comparación con los techos planos, mientras que los elementos de fachada como los balcones producen el efecto contrario.

El presente documento busca, aumentar el acervo de información, sobre los datos de la $\mathrm{Q}_{\mathrm{F}}$, en especial la $Q_{F v}$, para ser considerada a la hora de las simulaciones en los edificios que buscan su eficiencia energética y reflexionar tanto los pro y contras del diseño de un edificio, no solo en el interior sino en el exterior.

\section{Referencias}

ANSYS, Inc. (2013). ANSYS Fluent Theory Guide. USA: ANSYS, Inc.

Cengel , Y., \& Ghajar, A. (2011). Transferencia de calor y masa. Fundamentos y aplicaciones (4ta. ed.). Barcelona: McGrawHill.

Chapman, L., \& Thornes, J. (2005). The influence of traffic on road surface temperatures: implications for thermal mapping studies. Meteorological Applications, 12(4), 371-380.

Fan, H., \& Sailor, D. (2005). Modeling the impacts of anthropogenic heating on the urban climate of Philadelphia: a comparison of implementations in two PBL schemes. Atmos. Environ., $\quad 39(1), \quad 73-84$. doi:http://dx.doi.org/10.1016/j.atmosenv.2004.0 9.031

Flanner, M. (2009). Integrating anthropogenic heat flux with global climate models. Geophys Research Letters, 36(2), 2801.
Grajeda, R., Alonso, E., \& Esparza, C. (2018). Vehicular Anthropogenic Heat in the Physical Parameters of An Urban Canyon for Warm Humid Climate. 34th International Conference on Passive and Low Energy Architecture. 1, págs. 225-230. Hong Kong: PLEA.

Hernandez Sampieri, R., Fernandez Collado, C., \& Baptista Lucio, P. (2014). Metodología de la investigación (Sexta ed.). New York: Mc GrawHill.

Ichinose, T., Shimodozono, K., \& Hanaki, K. (1999). Impact of anthropogenic heat on urban climate in Tokyo. Atmosphere Anvironment, 33, 3897-3909.

Kolokotroni, M., Davies, M., Croxford, B., Bhuiyan, S., \& Mavrogianni, A. (2010). A validated methodology for the prediction of heating and cooling energy demand for buildings within the Urban Heat Island: Case-study of London. Solar Energy, 84(12), 2246-2255.

Koralegedara, S., Lin, C.-Y., Sheng, Y.-F., \& Kuo, C.-H. (2016). Estimation of anthropogenic heat emissions in urban Taiwan and their spatial patterns. Environmental Pollution, 215, 84-95.

Li, H., Zhou, Y., Li, X., Meng, L., Wang, X., Wu, S., \& Sodoudi, S. (2018). A new method to quantify surface urban heat island intensity. Science of the total environment(624), 262-272. doi:10.1016/j.scitotenv.2017.11.360

Llaguno-Munitxa, M., Bou-Zeid, E., \& Hultmark, M. (2017). The influence of building geometry on street canyon air flow: Validation of large eddy simulations against wind tunnel experiments. Journal of Wind Engineering and Industrial Aerodynamics, 165, 115-130. doi:10.1016/j.jweia.2017.03.007

Mirzaei, P. (2015). Recent challenges in modeling of urban heat island. Sustainable cities and sociaety, 19, 200-206.

Nazarian, N., \& Kleissl, J. (2015). CFD simulation of an idealized urban environment: Thermal effects of geometrical characteristics and surface materials. Urban climate, 12, 141159. doi:10.1016/j.uclim.2015.03.002 
Nazarian, N., \& Kleissl, J. (2016). Realistic solar heating in urban areas: Air exchange and streetcanyon ventilation. Building and Environment, 95, 75-93. doi:10.1016/j.buildenv.2015.08.021

Oke, T. (1988). The urban energy balance. Progress in Physical Geography, 12, 471-508.

Sailor, D. (2011). A review of methods for estimating anthropogenic heat and moisture emissions in the urban environment. Int. J. Climatol, 31(2), 189-199. doi:http://dx.doi.org/10.1002/joc.2106

Sailor, D., \& Lu, L. (2004). A top-down methodology for developing diurnal and seasonal anthropogenic heating profiles for urban areas. Atmospheric Environment, 38, 2737-2748.

Sailor, J., Georgescu, M., Milne, J., \& Hart, M. (2015). Development of a national anthropogenic heating database with an extrapolation for international cities. Antmospheric Environment, 118, 7-18.

Santamouris, M., Assimakopoulos, N., Chrisomallidou, N., Klitsikas, D., Mangold, P., \& Tsangrassoulis, A. (2011). Energy and Climate in the Urban Built Environment: The canyon effect. (M. Santamouris, Ed.) London \& New York: Routledge, Taylor \& Francis Group.

Stewart, I., \& Oke, T. (2012). Local climate zone for urban temperature studies. American Meteorology Sociaty, 93, 1879-1900.

Stromann-Andersen, J., \& Sattrup, P. (2011). The urban canyon and building energy use: Urban density versus daylight and passive solar gains. Energy and Buildings, 43(8), 2011-2020. 